\title{
Synthesis and mesomorphic properties of non-symmetric liquid crystalline dimers containing azobenzene groups
}

\begin{abstract}
Four novel nonsymmetric dimers containing azobenzene mesogenic groups were synthesized. The nonsymmetric dimers compounds namely, ethyl 4-[(4-\{4-(4-((4nitrophenyl)diazenyl)phenoxy)alkyloxy\}phenyl)diazenyl]benzoate were obtained from the alkylation of ethyl 4-[(4-(4-bromoalkyloxy)phenyl)diazenyl]benzoate with 4-[(4nitrophenyl)diazenyl]phenol. The mesomorphic properties of the compounds were determined by DSC and polarizing optical microscopy. The first member of the series was nonliquid crystalline while all other homologues display nematic and smectic A phases. The trans-azobenzene groups of the dimers display a high-intensity -* transition at about $365 \mathrm{~nm}$ and a low-intensity $\mathrm{n}^{*}$ transition at around $465 \mathrm{~nm}$, therefore, photochromism can be achieved by the introduction of the azo linkage to the dimeric liquid crystalline molecules.
\end{abstract}

Keyword: Mesophase; Azobenzene; Nonsymmetric dimmers; Cis-trans isomers; Nematic; Smectic 